\title{
SPECTROSCOPY OF QUASIMOLECULAR X-RAYS WITH H-LIKE DECELERATED IONS *
}

\author{
H. SCHMIDT-BÖCKING ${ }^{1)}$, R. SCHUCH ${ }^{2)}$, I. TSERRUYA ${ }^{3)}$, R. HOFFMANN ${ }^{2)}$, B. JOHNSON ${ }^{5)}$,
} K.W. JONES ${ }^{5)}$, E. JUSTINIANO ${ }^{2)}$, M. MĖRON ${ }^{5)}$, P.H. MOKLER ${ }^{6)}$, H. INGWERSEN ${ }^{4)}$, W. SCHADT ${ }^{1)}, M$. SCHULZ ${ }^{2)}$ and B. FRICKE ${ }^{\text {7) }}$

") Institut für Kernphysik, Johann-Wolfgang-Goethe Universität, 6000 Frankfurt am Main, Fed. Rep. Germany

${ }^{2)}$ II. Physikalisches Institut, Universität Heidelberg, 6900 Heidelberg, Fed. Rep. Germany

${ }^{3)}$ Weizmann Institute, Rehovot, Israel

4) MPI für Kernphysik, 6900 Heidelberg, Fed. Rep. Germany

5) Brookhaven National Laboratory, Upton, NY, USA

6) GSI-Darmstadt, 6100 Darmstadt, Fed. Rep. Germany

7) Gesamthochschule Kassel, 3500 Kassel, Fed. Rep. Germany

The quasimolecular $2 \mathrm{p} \pi-1 \mathrm{~s} \sigma$ transition energy as a function of the internuclear distance is obtained from the interference structure observed in quasimolecular $\mathrm{K} \mathrm{X}$-ray spectra from low energy $\mathrm{H}$-like projectiles measured at certain impact parameters.

It is well established that the formation of quasimolecular orbitals plays an important role in the excitation process in nearly symmetric ion-atom collisions [1]. The electronic excitation proceeds mainly by electron promotion via coupling of close lying quasimolecular states. The coupling strength is strongly dependent on the energy gap $\Delta E_{i f}$ between the quasimolecular orbitals $i$ and $f$, where $\Delta E_{i f}$ generally varies with the internuclear separation $(R)$ of both colliding nuclei. Therefore it is crucial for the theoretical description of the excitation process to have precise information on this energy difference as a function of $R$. For inner shells in heavy ion-atom collisions there are several ways to determine the quasimolecular binding energies as a function of the internuclear distance. Measuring the radiation emitted from the separated collision partners is one attempt to derive total or differential cross sections for excitation of different subshells and to compare these values with predictions made by theoretical models [2]. From this comparison indirect information on the $\Delta E_{i f}$ values can be obtained, which obviously depend on the accuracy (approximations) of the calculations. Furthermore, the experimental values mostly reflect the occupation long after the quasimolecular formation, when the vacancies decay in the separated collision systems. In addition, possible fast cascading processes in outer shells make a clean determination of the primary quasimolecular excitation and therefore of $\Delta E_{i f}$ rather difficult.

* Partiy supported by BMFT/FRG, the Minerva Foundation/FRG, Department of Energy/USA, and the DFG/ FRG.
It was expected [3] that the radiation emitted during the collision originating from transitions between quasimolecular states (quasimolecular radiation: MOR) would give a more direct access to these quasimolecular energy values $\Delta E_{i f}(R)$ than the radiation from separated systems. In the quasistatic approximation [4] the measured photon energy $E_{\mathrm{x}}$ is assumed to be identical with the binding energy difference $\Delta E_{i f}$ of the two participating orbitals. Here MO X-rays with energy $E_{x}$ can be emitted only at a given internuclear distance $R\left(E_{\mathrm{x}}\right)$. For a fixed impact parameter $b$, the emission probability $\Delta P_{i f}\left(b, E_{\mathrm{x}}\right) / \Delta E_{\mathrm{x}}$ per photon energy interval (assuming that the orbital has one vacancy) is then given by:

$$
\frac{\Delta P_{i f}\left(b, E_{\mathrm{x}}\right)}{\Delta E_{\mathrm{x}}}=2 \frac{\Delta R\left(E_{\mathrm{x}}\right)}{v_{\mathrm{R}}} A\left(E_{\mathrm{x}}\right),
$$

where $v_{\mathrm{R}}$ is the radial component of the ion velocity and $\Delta R / v_{\mathrm{R}}$ corresponds to that time interval in which photons in the energy range $E_{\mathrm{x}}$ to $E_{\mathrm{x}}-\Delta E_{\mathrm{x}}$ can be emitted. $A\left(E_{\mathrm{x}}\right)$ denotes the radioactive transition rate. The factor of 2 reflects the possibility of a decay both on the incoming as well as on the outgoing part of the trajectory.

In the following we will discuss only transitions into the quasimolecular $f=1 s \sigma$ orbital, the innermost quasimolecular state. $(i=2 \mathrm{p} \pi$ or $2 \mathrm{po})$. Within the framework of the quasimolecular picture at a given $b$ the photon energy for 1s $\sigma$ MOR extends from the $K_{\alpha / \beta}$ line of the separated systems up to the energy difference $\Delta E_{i f}\left(R_{\min }\right)$ between the orbitals $i$ and $f$ at the distance of closest approach $R_{\min }$. Using eq. (1) and calculated transition rates, $\Delta E_{i f}(R)$ could thus be derived from 
measured continuous MOR probabilities. Numerous experiments and theoretical studies of the 1s $\sigma$ MOR have shown, however, that the quasi-static approximation is a rather crude one and not appropriate for describing MOR spectra in ion-atom collisions [5].

A more precise investigation of the dynamics of MOR shows that the photon energy $E_{\mathrm{x}}$ is no longer identical with $\Delta E_{i f}(R)$. Any excess or deficit of $\mathrm{X}$-ray energy can be obtained from the conversion of translational kinetic energy of the two colliding nuclei into photon energy, giving rise to the so-called collision broading. Furthermore, in nearly all experimental investigations so far "lowly charged" projectiles were used where 1so MOR could be emitted only if the 1 so vacancy production $a_{f}(t)=a_{1 s o}(t)$ is strongly dependent on the collision time and which were also rather difficult to calculate. Weisskopf [6] has shown that the spectrum for such a transition $i \rightarrow f$ can be calculated from the Fourier transform of the time-dependent dipole matrix element $D_{i f}(R(t))$ (see also ref. [7]).

Taking the dynamics of the collision into account one obtains for the emission probability

$$
\frac{\Delta P_{i f}\left(b, E_{\mathrm{x}}\right)}{\Delta E_{\mathrm{x}}} \sim \mid \int_{-\infty}^{+\infty} \mathrm{d} t \cdot a_{f}(R) D_{i f}(R)
$$

$$
\times\left.\exp \left\{\frac{\mathrm{i}}{\hbar} \cdot \int_{0}^{t}\left[E_{\mathrm{x}}-E_{f}(R)+E_{i}(R)\right] \mathrm{d} t^{\prime}\right\}\right|^{2},
$$

where $R=R(b, t)$ is dependent on $b$ and $t$. A detailed description of the dynamical theory is given in ref. [5].

The spectral shape of the 1s $\sigma$ MOR for a given impact parameter is in clear contradiction to the quasistatic prediction but in agreement with this dynamical theory. The spectrum extends further than the maximal quasistatic transition energy at the distance of closest approach showing an exponential slope beyond this $\mathrm{X}$-ray energy. The MOR spectrum is bare of any significant structure (see fig. 1) and does not allow a reliable assignment between the measured photon energies $E_{\mathrm{x}}$ and $\Delta E_{i f}(R)$.

In conclusion, from the measured MOR emission probabilities for low-charged fast ions, where the 1 s $\sigma$ vacancy is created at small $R$ in the same collision, no quasimolecular spectroscopy could be performed so far. This result indeed is disappointing after the tremendous amount of work which has been done on this field [5] in the last decade.

There is, however, an access to a reliable quasimolecular spectroscopy for the the $1 \mathrm{~s} \sigma$ orbital if $\mathrm{H}$-like ions are used as projectiles for these investigations. For

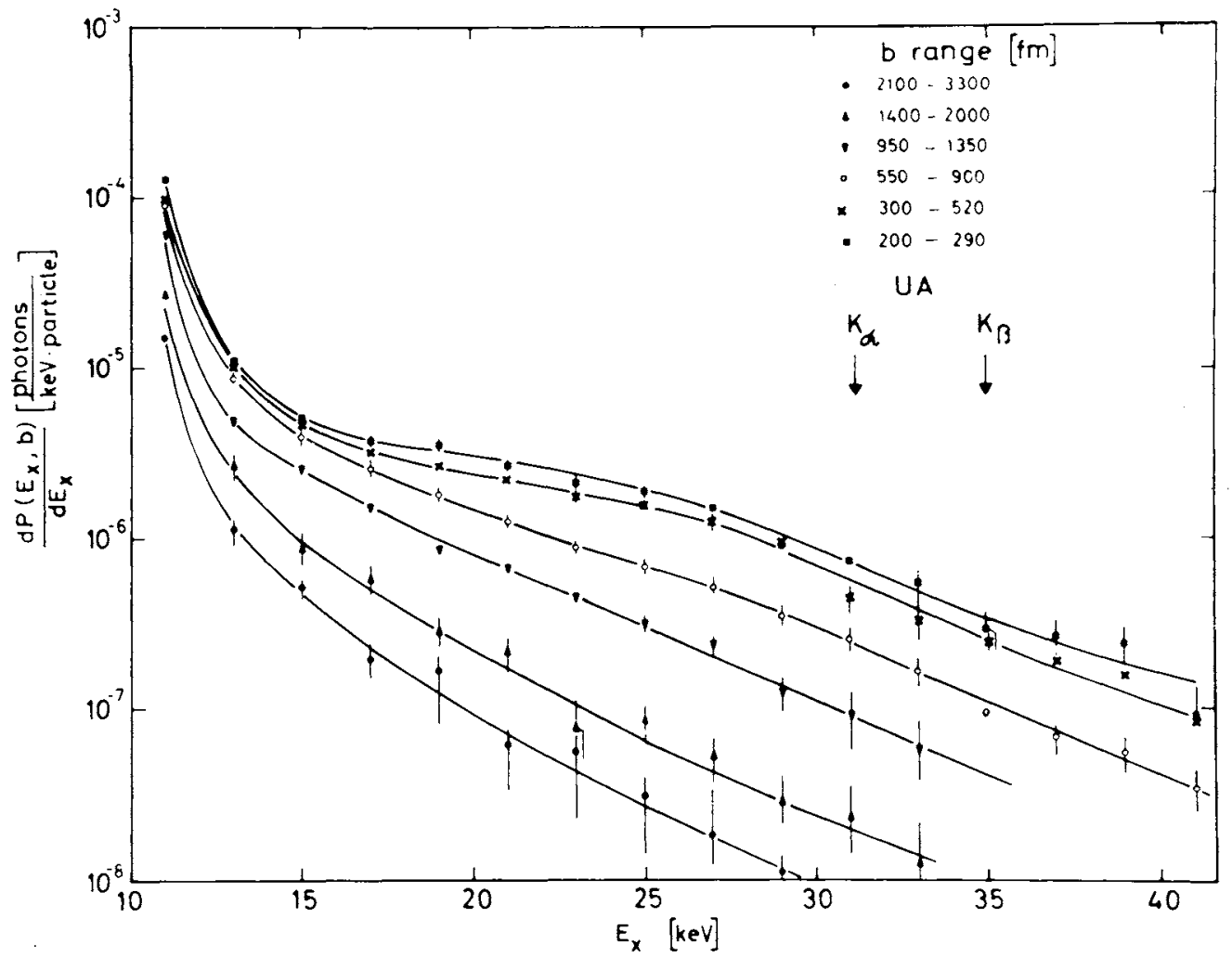

Fig. 1. MOR-emission probabilities as a function of X-ray energy $E_{\mathrm{x}}$ and impact parameter $b$ for $90 \mathrm{MeV} \mathrm{Ni}$ on $\mathrm{Ni}$. The solid lines are to guide the eye. 
H-like projectiles the transition to a given $E_{\mathrm{x}}$ therefore can occur on the incoming as well as on the outgoing part of the trajectory ("way-in" and "way-out").

These two transition amplitudes cannot be distinguished experimentally. Since for such slow heavy-ion collisions both amplitudes have a well defined phase relation they will interfere. For appropriate velocities the experimental spectrum should show a significant interference structure.

This interference structure allows a direct determination of the relevant phase differences which can be used for a determination of the energy gap of the involved orbitals $i$ and $f$.

In fig. 2 the "two way" MOR decay process is illustrated. As a function of $R$ the two relevant quasimolecular orbitals are shown. For low velocities the transition with photon energy $E_{\mathrm{x}}$ will occur at $R_{-}\left(E_{\mathrm{x}}\right.$, $\left.-t_{0}\right)$ and $R_{+}\left(E_{\mathrm{x}},+t_{0}\right)$. The transition amplitudes on the "way-in" $\left(-t_{0}\right)$ and "way-out" $\left(+t_{0}\right)$ are qualitatively shown in the lower part of fig. 2 . With decreasing velocity (see stationary phase approximation $[5,8]$ photons with $E_{\mathrm{x}}$ will be emitted only in the region around $R_{-}$and $R_{+}$. The phase difference of the transition amplitude at $-t_{0}$ and $+t_{0}, \Delta \phi\left(E_{\mathrm{x}}\right)$, can be determined from the time evolution of the wave functions of both involved molecular orbitals and of the dipole operator. Using the stationary phase approximation it can be shown that the coherent addition of these two amplitudes with the phase difference $\Delta \phi\left(E_{\mathrm{x}}\right)$ leads to an interference term in the emission probability which

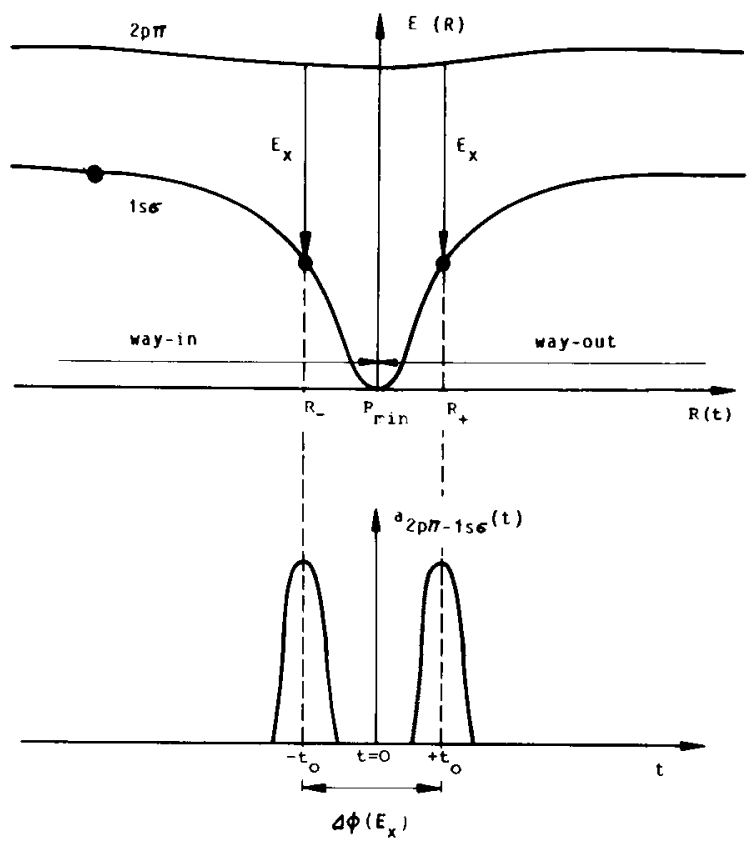

Fig. 2. Illustration of the "two-way" MO decay process with H-like projectiles. causes an oscillation in the shape of the MOR spectrum. This oscillation can be de scribed by $[8,9]$

$$
\frac{\Delta P_{i f}\left(b, E_{\mathrm{x}}\right)}{\Delta E_{\mathrm{x}}} \sim \cos ^{2}\left(\frac{\Delta \phi\left(E_{\mathrm{x}}\right)}{2}-\frac{\pi}{4}\right),
$$

where the phase difference $\Delta \phi_{i f}$ is

$\Delta \phi_{i f}\left(E_{\mathrm{x}}\right)=\frac{2}{\hbar} \int_{0}^{t_{0}}\left[E_{\mathrm{x}}-\Delta E_{i f}(R)\right] \mathrm{d} t^{\prime}$.

The constant phase term $\pi / 4$ is explained in refs. [8,9]. From the measured spectra, experimental $\Delta \phi$ values as a function of $E_{\mathrm{x}}$ can now be determined. Using eq. (4) a simple quantitative relation between $E_{\mathrm{x}}$ and $R$ can now be derived. In eq. (4), however, all $R_{\min } \leqslant R \leqslant R\left(E_{\mathrm{x}}\right)$ contribute to the phase integral making the assignment between $E_{\mathrm{x}}$ and $R\left(E_{\mathrm{x}}\right)$ rather difficult. However, from the derivative of $\delta \Delta \phi / \delta E_{\mathrm{x}}$ in a simple direct way $E_{\mathrm{x}}=$ $E_{\mathrm{x}}(R)=\Delta E_{i f}(R)$ can be determined. This derivative yields

$$
\frac{\delta \Delta \phi\left(E_{\mathrm{x}}\right)}{\delta E_{\mathrm{x}}} \approx \frac{2}{\hbar} t_{0}\left(E_{\mathrm{x}}\right)
$$

where $2 t_{0}$ is the transit time along the classical trajectory from the position $-R\left(E_{\mathrm{x}}\right)$ to $+R\left(E_{\mathrm{x}}\right)$. The transit time can be easily converted into the path length $S$ or into the internuclear separation $R\left(E_{\mathrm{x}}\right)$ assuming a Rutherford trajectory. Simple measurements of the oscillatory structure of the MOR spectra at a given impact parameter $b$ therefore yield the X-ray energy dependence of $\Delta \phi$ and from this in a straightforward way $\Delta E_{i f}$ can be determined, i.e. spectroscopic information on these transiently formed quasimolecular orbitals can be obtained only on the basis of quantummechanically well established phase relationships. For the observation of at least one or more oscillations in the MOR emission probabilities, $\mathrm{H}$-like projectiles are needed with velocities $v_{\mathrm{p}} \leqslant 0.2 v_{\mathrm{K}}^{\mathrm{UA}}$ where $v_{\mathrm{K}}^{\mathrm{UA}}$ is the $\mathrm{K}$ electron velocity in the united atom (UA) system. Since presently no ion sources for H-like ions $\left(Z_{\mathrm{p}} \geqslant 16\right)$ with sufficient intensity in combination with suitable accelerators are available, the only way to produce such beams is the so-called accel-stripping-decel technique [10-12]. Ions are accelerated to such high velocities that the penetration of a thin $\mathrm{C}$-foil produces a considerable fraction of one-electron ions ( $\mathrm{H}$-like). These ions are then decelerated to such low velocities that the above mentioned condition is fulfilled. One of the very well working accel-decel systems is the 4-stage-tandem facility of the Brookhaven National Laboratory, where the $\mathrm{Cl}^{16+}$-on-Ar collision system was investigated [10]. The $\mathrm{S}^{15+}$-on-Ar system was measured with the tandempostaccelerator system of the MPI für Kernphysik in Heidelberg [11]. Very recently the $\mathrm{Ge}^{31+}$-on-Kr system was investigated at the Unilac of GSI-Darmstadt [12].

To measure the MOR emission probabilities for a given $b$, the emitted photon $\left(\rightarrow E_{\mathrm{x}}\right)$ and the scattered 


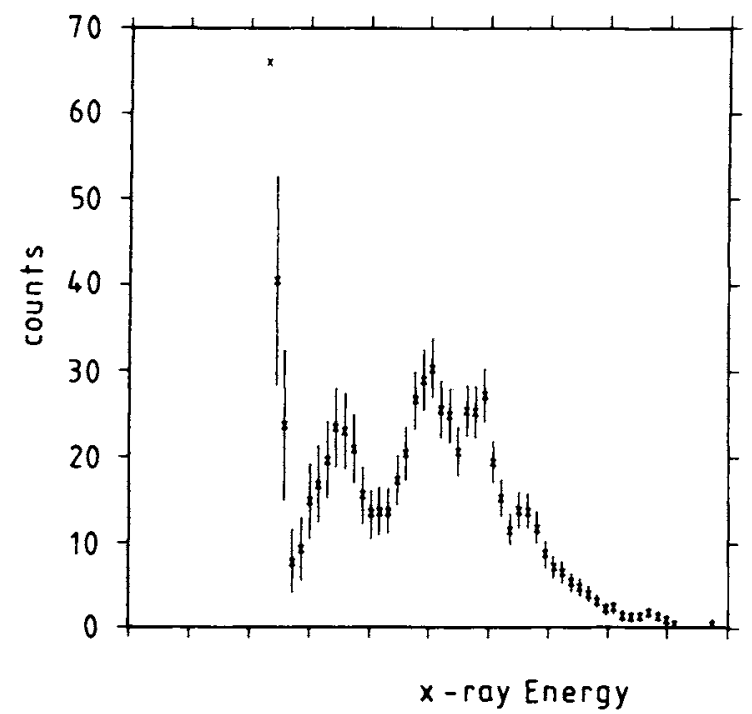

Fig. 3. Coincident absorber-corrected 1so MOR spectra for 2.5 $\mathrm{MeV} \mathrm{Cl}{ }^{16+}$ on Ar.

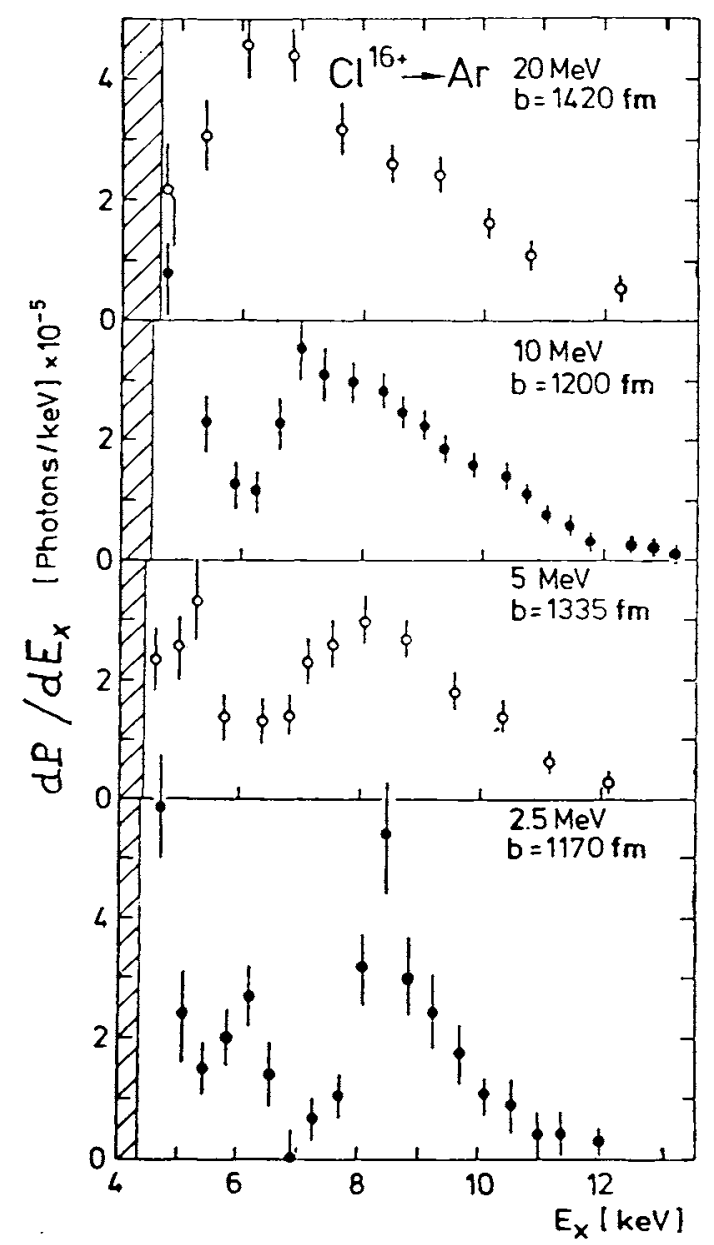

Fig. 4. Projectile velocity dependence of absolute 1 s $\sigma$ MOR emission probabilities for $\mathrm{Cl}^{16+}$ on Ar. projectile $(\rightarrow b)$ have to be detected in coincidence. The well collimated H-like beam $\left(\sim 10^{8}\right.$ ions/s) hit a differentially pumped gas target where the pressure was kept low enough to avoid charge exchange of the beam before entering the central target area. The scattered projectiles were detected with a position sensitive parallel plate avalanche detector and the X-rays with a $\mathrm{Si}(\mathrm{Li})$ detector. The coincidence electronic is presented in ref. [13]. The data were collected in "event mode". Because of the low beam intensity and target density the contributions of random coincidences in the MOR regime were negligible. In fig. 3 an absorber-corrected coincidence spectrum for $\mathrm{Cl}^{16+}$ on $\mathrm{Ar}$ is shown. In contradiction to fig. 1 a clear oscillatory structure is observed. Because of the experimental difficulties (the true coincidence rate is 1 per $10 \mathrm{~min}$ ) and limited beam time the statistical error could not be further reduced. For the same system in fig. 4 the velocity dependence of this structure is presented. With decreasing velocity $\Delta \phi$ increases and more structure appears, in nice agreement with the scaling of the stationary phase approximation $\left(\Delta \phi \sim t \sim 1 / v_{\mathrm{p}}\right)$. Even for $\mathrm{Ge}^{31+}$ on $\mathrm{Kr}$ the oscillatory structure in the emission probability $\Delta P / \Delta E_{\mathrm{x}}$ has been observed [14].

In fig. 5 for $\mathrm{Cl}^{16+}$ on $\mathrm{Ar}$ for $2.5,5,10$ and $20 \mathrm{MeV}$ the positions of constructive (maxima) and destructive (minima) interferences are shown. They follow nicely the scaling with $v_{\mathrm{p}}$ and $b$ of the stationary phase approximation. Fig. 6 depicts for $b \approx 1000 \mathrm{fm}$ the experimental phases times the velocity $\left(\Delta \phi \cdot v_{\mathrm{p}}\right)$ for constructive and destructive interferences (maxima and minima from fig. 5). In agreement with eq. (4) they scale well on

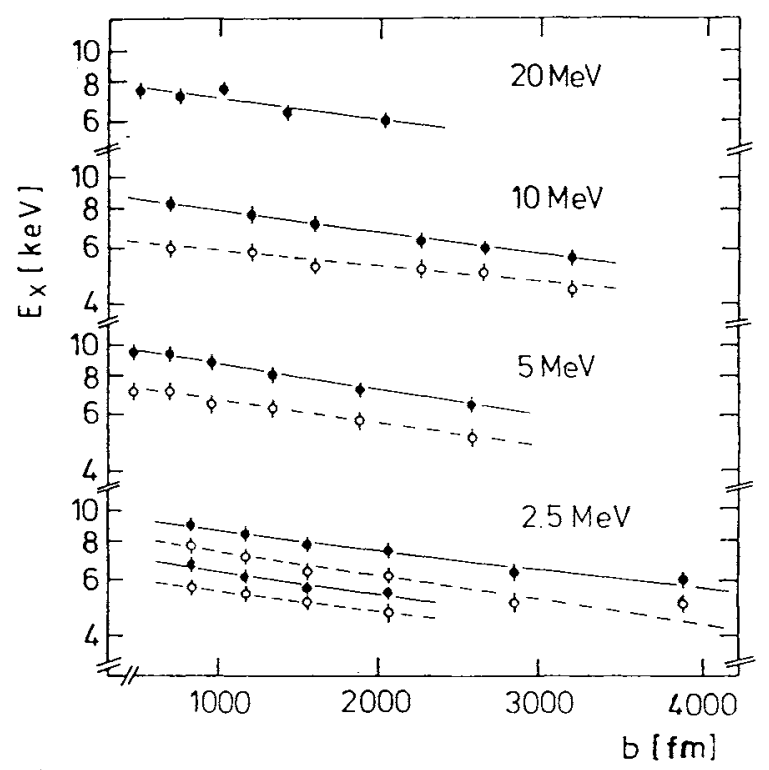

Fig. 5. X-ray energies for constructive (solid lines) and destructive (dashed lines) interferences for $\mathrm{Cl}^{16+}$ on $\mathrm{Ar}$ as a function of projectile velocity and impact parameter. 


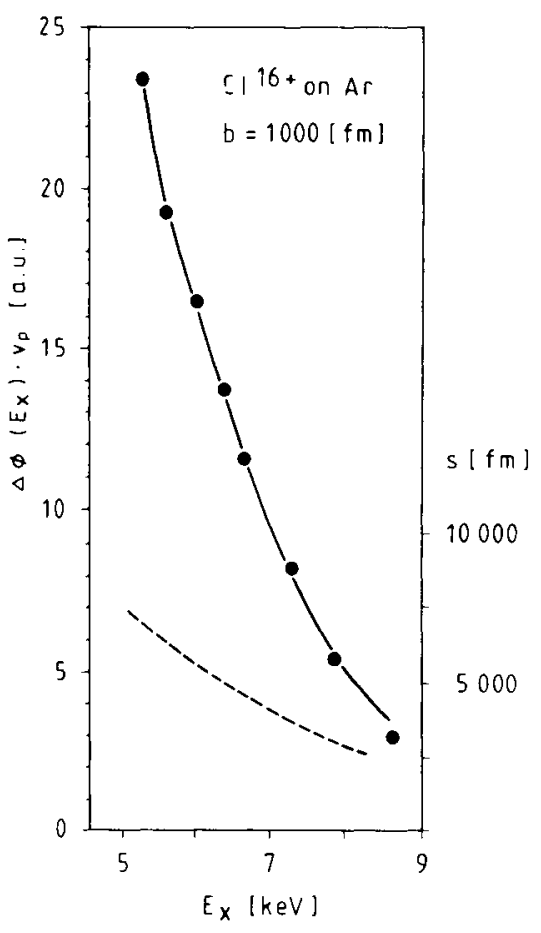

Fig. 6. Experimentally determined phase differences times projectile velocity $\left(\Delta \phi \cdot v_{\mathrm{p}}\right)$ (solid line). The dashed line represents the derivative of the path length (see text).

one common curve. The derivative of this experimental curve yields directly the path length $S\left(E_{\mathrm{x}}\right)$. From this function $S=S\left(E_{\mathrm{x}}\right)$ the $\Delta E_{i f}(R)$ can be immediately determined.

Using only the "clean" experimental information on the positions of the maxima and the minima we obtain for eq. (5) the following approximation:

$\delta \Delta \phi(\max -\min )=\pi=\delta E_{\mathrm{x}}(\max -\min ) \frac{2}{\hbar} t_{0}\left(E_{\mathrm{x}}\right)$.

The measured $\delta E_{\mathrm{x}}(\max -\min )$ can be taken from fig. 5 . With these data the corresponding $t_{0}\left(E_{\mathrm{x}}\right)$ values can be calculated and can then be converted into the internuclear separation $R\left(E_{\mathrm{x}}\right)$ using a classical Coulomb trajectory. The so obtained $E_{\mathrm{x}}=E_{\mathrm{x}}(R)$ values are presented in fig. 7 for $\mathrm{Cl}^{16+}$ on Ar. Independent of the projectile velocity they all scale on a common curve in agreement with the theory. The so derived transition energies are compared in fig. 7 with calculated $\mathrm{MO}$ transition energies, using a two center potential Dirac-Fock program for 26 electrons in the $\mathrm{Cl}-\mathrm{Ar}$ collision system [15]. The solid line represents $2 \mathrm{po}-1 \mathrm{~s} \sigma$ and the dashed line $2 \mathrm{p} \pi-1 \mathrm{~s} \sigma$ transitions. It can be seen that the calculated $2 \mathrm{p} \pi-1 \mathrm{~s} \sigma$ transition energies qualitatively agree with the measured $R$ dependence but are slightly above the experimental values, even though the screening is somewhat overestimated. The predicted val-

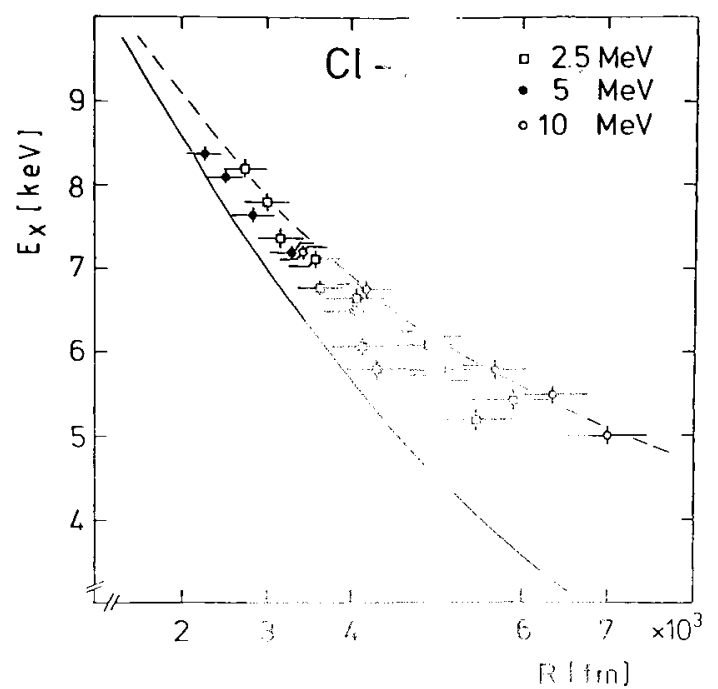

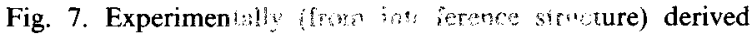
$2 \mathrm{p} \pi-1 \mathrm{~s} \sigma$ energy dffrences a whon of the internuclear separation. The curses restesent t? aroical collotations of the $2 s \sigma-1 s \sigma$ (solid line) and the $2 m-1 s a$ (dashed line) energy difference (see tex?

ues for 2po-1 wa tranitions are thatw beswe the experimental values. This jesth is in agroment with the expected intensity combibitions of both transitions into the 1 s $\sigma$ state [9].

In conclusion for H-ijke ions $\$^{5}$ and $\mathrm{Cl}^{15+}$ on $\mathrm{Ar}$ and for $\mathrm{Ge}^{\text {it }}$ on $\mathrm{K}$. the experimental is o MOR spectra $[9,14]$ show a clear osulatery structure originating from the interterence of the travition amplitudes on the incorming and ougoing parts of the trajectory.

From the interforence structure information on the phase relationshion of ines shull trasition amplitudes can be obtanes. From the sexpermentaliy determined phase differences, quasimolecular 2 pr-1s r ransition

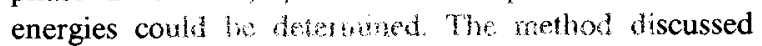
above presents a mique onsibility for spectoscopy of quasimolecular onhtats ant mighs wen be applicable in

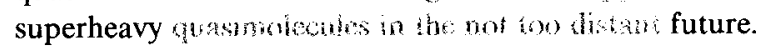

\section{References:}

[1] Q.C. Kossel, Case Smores in Aromig Physics. Vol. 1, eds.,

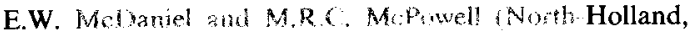

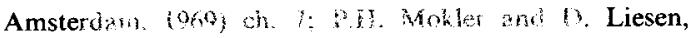
Progress in Atomic spoctroscopy nay 6 , eas. P.F. Beyer and H. Keinpoppen (Plenum, rew York, 1984) p. 321.

[2] F. Bosch, P. Armbruster, D. Liesen, D. Mawi, P.74. Mokler, H. Schmid Bocking and R. Guat. 2. Phys. A296 (1980) 11; D. Insen. P. Armbrusier, Fosch, PH. Mokler, H. Schmid-Boking Re Sonch. I.R. Witheiny ane H.J. Wollersheim. Phys Rery lent \&A (1980) ox?

[3] F.W. Saris, W.F. van der Weg. H. Tawata and R Laubert, 
Phys. Rev. Lett. 28 (1972) 717; P.H. Mokler, H.J. Stein and P. Armbruster, Phys. Rev. Lett. 29 (1972) 827.

[4] J.S. Briggs J. Phys. B7 (1974) 47; W.E. Meyerhof, T.K. Saylor, S.M. Lazarus, A. Little, B.B. Triplett, L.F. Chase and R. Anholt, Phys. Rev. Lett. 32 (1974) 1279.

[5] R. Anholt, Rev. Mod. Phys., to be published.

[6] V.F. Weisskopf, Phys. Z, 34 (1933) 1.

[7] R. Anholt, Z. Phys. A288 (1978) 257.

[8] J.H. Macek and J.S. Briggs, J. Phys. B7 (1974) 1312.

[9] I. Tserruya, R. Schuch, H. Schmidt-Böcking, J. Barrette, Wang Da-Hai, B.M. Johnson, K.W. Jones and M. Mèron, Phys. Rev. Lett. 50 (1983) 30; R. Schuch et al., Z. Physik, to be published.
[10] P. Thieberger, J. Barette, B.M. Johnson, K.W. Jones, M. Mèron and H.E. Wegener, IEEE Trans. Nucl. Sci. NS-30 (1983) 1431.

[11] H. Ingwersen, E. Jaeschke and R. Repnow, Nucl. Instr. and Meth. 215 (1983) 55.

[12] P.H. Mokler, D.H.H. Hoffmann, W.A. Schönfeldt, P. Maor, W.E. Meyerhof and Z. Stachura, Nucl. Instr. and Meth. B4 (1984) 37.

[13] R. Hoffmann, G. Gaukler, G. Nolte, H. Schmidt-Böcking and R. Schuch, Nucl. Instr. and Meth. 197 (1982) 391.

[14] R. Schuch, private communication.

[15] B. Fricke, T. Morović and W.-D. Sepp, private communication. 